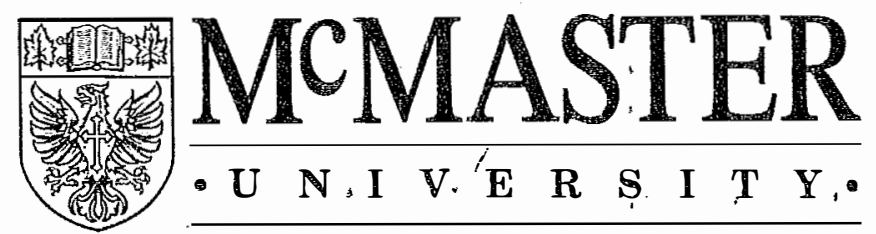

M I C H A E L G. D E G R O O T E SCHOOL OF BUSINESS

\section{RESEARCH AND WORKING PAPER SERIES}

\title{
LOT STREAMING WITH DETACHED SETUPS IN THREE-MACHINE FLOW SHOPS
}

By

\section{liang Chen and George Steiner}

Management Science and Information Systems Area

Michael G. DeGroote School of Business

McMaster University,

Hamilton, Ontario, Canada

Working Paper \# 399

January, 1995

Innis

\section{TTER UNIVERSITY}

$\mathrm{HB}$

in Street West

1, Ontario, Canada L8S 4M4

74.5

.R47 


\title{
LOT STREAMING WITH DETACHED SETUPS IN THREE-MACHINE FLOW SHOPS
}

\author{
By \\ Jiang Chen and George Steiner \\ Management Science and Information Systems Area \\ Michael G. DeGroote School of Business \\ McMaster University, \\ Hamilton, Ontario, Canada
}

Working Paper \# 399

January, 1995 


\title{
Lot Streaming with Detached Setups in Three-Machine Flow Shops*
}

\author{
Jiang Chen and George Steiner \\ Management Science and Information Systems Area \\ McMaster University \\ Hamilton, Ontario, Canada
}

\begin{abstract}
Lot streaming is the process of splitting a job or lot to allow overlapping between successive operations in a multistage production system. This use of transfer lots usually results in a shorter makespan for the corresponding schedule. In this paper, we study the structural properties of schedules which minimize the makespan for a single job with detached setup times in a flow shop. Although the structure of the optimal schedules is more complex than in the case with no setups [6], it is possible to find the optimal solution with $s$ sublots in $O(\log s)$ time for the three-machine case.
\end{abstract}

Keywords: Scheduling, flow shop, lot streaming.

\section{Introduction}

Lot streaming is the process of using transfer batches to move the processed portion of a production lot to downstream machines so that the makespan of the schedule can be shortened and the work-in-process inventory levels can be lowered. The term was introduced by Reiter[16], but the idea has

* This research was supported in part by the Natural Sciences and Engineering Research Council of Canada under Grant No. A1798. 
been considered many times under different names. The increased interest in its applications over the last few years is probably due to the fact that it is consistent with the Just-In-Time (JIT) philosophy of making small or single unit sublots and it also agrees with the basic idea of the OPT scheduling package [5], [7].

Szendrovits[17] analyzes the lot streaming problem in a flow shop for a single job with equal sublot sizes. Goyal [8] finds the optimal sublot sizes in Szendrovits' model. Moily[12], Jacobs and Bragg [10], Kulonda [11] and Graves and Kostreva [9] also demonstrate reductions in production time and cost by using transfer lots. Steiner and Truscott [18] find the optimal lot streaming schedules in an open shop with equal size transfer lots and no idling on the machines. Cetinkaya and Gupta [3] analyze the lot streaming problem for a single job in a flow shop with the total flow time criterion.

Most papers on lot streaming consider the objective of minimizing the makespan in an $m$-machine flow shop where each item is processed on the $m$ machines in the order $1, \ldots, m$. Trietsch, in [19] and [20], and Baker [1] independently develop a conceptual framework for the problem. They present a classification scheme and review the most important results in [21]. Vickson [22] solves the lot streaming problem for multiple jobs in a two-machine flow shop with job setup times and sublot transfer times.

In this paper, we consider the problem of minimizing the makespan by splitting a single job into $s$ sublots in an $m$-machine flow shop, where the job requires a detached setup on each machine. A setup is detached (or anticipatory) if it can be performed as soon as the machine is available. We use the more frequently used assumption of batch availability, i.e., items become available for processing at the next machine after the current machine finished processing the last item in their sublot (batch). More formally, we have $m$ machines, denoted by $M_{1}, M_{2}, \ldots, M_{m}$, the job has positive processing times $p_{1}, p_{2, \ldots,} p_{m}$ and detached setup times $S_{1}, S_{2}, \ldots, S_{m}$ on $M_{1}, M_{2}, \ldots, M_{m}$, respectively. If $x_{i, j}(i=1, \ldots, m, j=1, \ldots, s)$ is the size of the $j$ th sublot on $M_{i}$, then our objective is to find the $x_{i, j}$ values which minimize the makespan. We assume that the sublot sizes are normalized to represent the corresponding proportion of the job, i.e., $\sum_{j=1}^{s} x_{i, j}=1$ for $i=1, \ldots, m$. Thus, the processing time of sublot $j$ on $M_{i}$ is $p_{i} x_{i, j}$. The sublots are consistent if $x_{i, j}=x_{i+1, j}$ for $i=1, \ldots, m-1, j=1, \ldots, s$, otherwise they are variable. For consistent sublots we can write $x_{j}$ instead of $x_{i, j}$.

Another, less frequently studied lot streaming model uses the assumption 
of item availability when individual items become available for processing at the next machine as soon as they are finished on the current machine (unit size transfer lots). Vickson and Alfredsson [23] solve the makespan minimization problem with no setups in the two machine flow shop. The same problem is solved with detached setups in [4] and with attached setups in [2].

Most analytical results using the model with batch availability apply to flow shops with no setups, with the exception of the two-machine case [22]. Baker [1] shows that linear programming can be used to find the consistent sublot sizes which minimize the makespan. As Glass et. al. [6] point out, however, the linear programming approach provides little insight into the structure of the solution which would enable more general models to be solved. Potts and Baker [14] show that for a single job, it is sufficient to consider identical sublot sizes on the first two machines, and on the last two machines. The $m=2$ case is solved in [14] and in [19]. Glass et. al. [6] develop the solution to minimize the makespan for a single job in a three stage production process without setup times. Their algorithms compute the minimum makespan in $O(\log s)$ time for both the flow shop and job shop problem. They also present some structural results for $m>3$. In this paper, we generalize these results for flow shops with detached job setups. The presence of setups makes the structure of the optimal solution substantially more complex in most cases. Nevertheless, it is possible to extend most of the results from [6] to our case. For the three-machine flow shop, this leads to an algorithm which finds the optimal schedule in $O(\log s)$ time.

The paper is organized as follows. Section 2 develops some fundamental structural results and introduces reduced versions of the problem, which are simpler but equivalent to the original one. Section 3 gives a detailed analysis of the three-machine network. Section 4 presents the algorithms for solving the problem on three machines. The computation time required is $\mathrm{O}(\log \mathrm{s})$. A summary and conclusions are presented in Section 5.

\section{Networks and Fundamental Results}

In this section, we study first the relationships between optimal sublot sizes on different machines, which reveals that the sublot sizes could be made consistent on the first two and the last two machines. By further simplifying 
the problem, we show that it is equivalent to an alternative problem with no setups on $M_{1}$ and $M_{m}$.

Let $C_{i, j}$ denote the completion time of sublot $j$ on machine $i(i=$ $1,2, . ., m, \quad j=1,2, \ldots, s)$. The following constraints must be satisfied by any feasible solution.

1) Machine capacity constraints :

$$
C_{i, j} \geq C_{i, j-1}+p_{i} x_{i, j} \quad(i=1,2, \ldots, m, \quad j=2, \ldots s)
$$

2) Production constraints :

$$
C_{i, j} \geq C_{i-1, h(i, j)}+p_{i} x_{i, j} \quad(i=2, \ldots, m, \quad j=1,2, \ldots, s),
$$

where $h(i, j)$ is the last sublot on machine $i-1$ containing items included in sublot $j$ on machine $i$;

3) Initialization constraint :

$$
C_{i, 1} \geq S_{i}+p_{i} x_{i, 1} \quad(i=1,2, \ldots, m) .
$$

Theorem 1 There exists an optimal schedule in which $x_{1, j}=x_{2, j}$ and $x_{m, j}=$ $x_{m-1, j}$ for $j=1,2, \ldots, s$.

Proof. Suppose there is an optimal schedule $\pi$, in which the sublot sizes on the first two machines, $x_{i, j} i=1,2, j=1,2, \ldots, s$ are not consistent. We construct a schedule $\pi^{\prime}$ which is no worse than schedule $\pi$ with respect to the makespan and has consistent sublot sizes on the first two machines. Let $x_{i, j}^{\prime}$ denote the size of sublot $j$ on $M_{i}$ in the schedule $\pi^{\prime}$.

First, keep sublot sizes on $M_{2}, M_{3}, \ldots, M_{m}$ the same as in the schedule $\pi$, i.e., $x_{i, j}^{\prime}=x_{i, j} \quad(i=2,3, \ldots, m)$. Second, let sublot $k$ be the first sublot such that $x_{1, k} \neq x_{2, k}$, and we set the sublot sizes of schedule $\pi^{\prime}$ on $M_{1}$ using the sublots from $\pi$ :

$x_{1, j}^{\prime}=x_{1, j}$ for $j=1,2, \ldots, k-1$ and $x_{1, j}^{\prime}=x_{2, j}$ for $j=k, \ldots, s$.

Let $t_{i, j}$ and $t_{i, j}^{\prime}$ denote the starting times of the $j$ th sublot on $M_{i}(i=$ $1,2)$ in the two schedules, respectively. We claim that $t_{i, j} \geq t_{i, j}^{\prime}$ for $j=$ $1, \ldots, s$. For $j=1, \ldots, k-1$, this is obvious. If $x_{1, k}>x_{2, k}$, then from $x_{1, k}^{\prime}=x_{2, k}$ it follows that $C_{1, k}^{\prime}<C_{1, k}$, implying $t_{2, k}^{\prime} \leq t_{2, k}$. If $x_{1, k}<x_{2, k}$, then $h(2, k)>k$ and $\mathrm{C}_{1, h(2, k)} \leq t_{2, k}$ by the production constraints, so enlarging $x_{1, k}$ to $x_{1, k}^{\prime}=x_{2, k}$ will result in $C_{1, k}^{\prime} \leq C_{1, h(2, k)} \leq t_{2, k}$. Thus, the larger lot size $x_{1, k}^{\prime}$ will not delay beyond $t_{2, k}$ the start of the $k$ th sublot on $M_{2}$, i.e., $t_{2, k}^{\prime} \leq t_{2, k}$. A similar argument can be used to show inductively that $t_{2, j} \geq t_{2, j}^{\prime}$ for every $j>k$. 
So no sublot starts later in $\pi^{\prime}$ than in $\pi$ on $M_{2}$. Since $x_{2, j}=x_{2, j}^{\prime}$ for $j=1, \ldots, s$, no sublot finishes later in $\pi^{\prime}$ than in $\pi$ on $M_{2}$. So $\pi^{\prime}$ is at least as good a schedule as $\pi$.

To prove that there is an optimal schedule $\pi^{n}$ with consistent sublots on $M_{m-1}$ and $M_{m}$, we can make the sublots on $M_{m}$ the same as on $M_{m-1}$, i.e., define $x_{m, j}^{\prime \prime}=x_{m-1, j}$ for $j=1, \ldots, s$ and a similar argument to the one above proves that $\pi^{n}$ is also optimal.

Corollary 2 For the two- and three-machine problem there exists an optimal schedule with consistent sublots.

We note that Corollary 2 does not extend to the case of four or more machines. This was demonstrated by an example in [14], even without setup times.

The following lemmas can further simplify the original problem.

Lemma 3 If $C_{\max }^{1}$ denotes the optimal makespan for the alternative lot streaming problem (A1) in which the setup times $S_{i}$ are replaced by $\max \left\{S_{i}-\right.$ $\left.S_{1}, 0\right\}(i=1,2, \ldots, m)$, then $C_{\max }=C_{\max }^{1}+S_{1}$ and the optimal sublot sizes for $(A 1)$ are also optimal for the original problem.

Proof. Since the setups are detached, we can assume that all machines are being set up during the setup of $M_{1}$ in the interval $\left[0, S_{1}\right]$. Thus, our lot streaming problem is equivalent to an alternative problem in which no setup may start before $t=S_{1}$ and the $i$ th setup time has been reduced to $\max \left\{S_{i}-S_{1}, 0\right\}(i=1,2, \ldots, m)$.

Lemma 4 Consider an alternative lot streaming problem (A2) in which the setup time on $M_{m}$ is replaced by 0 and let $C_{\max }^{2}$ be the optimal makespan for this problem. Then $C_{\max }=\max \left\{C_{\max }^{2}, p_{m}+S_{m}\right\}$ and the optimal sublot sizes for $(A 2)$ are also optimal for the original problem.

Proof. Let $I_{m}$ be the total idle time on $M_{m}$ in the optimal schedule for the alternative problem $(A 2)$. W.l.o.g. we can assume that all sublots are scheduled on $M_{m}$ as late as possible without increasing the makespan. Thus, $t=I_{m}$ represents the start time for processing the first sublot on $M_{m}$ in this schedule and $C_{\max }^{2}=I_{m}+p_{m}$. We clearly must have $C_{\max } \geq C_{\max }^{2}$ and 
$C_{\max } \geq S_{m}+p_{m}$. Therefore, if $S_{m} \leq I_{m}$, then we can finish setting up $M_{m}$ before $t=I_{m}$ for the original problem and use the optimal schedule obtained for $(A 2)$. This must result in an optimal schedule for the original problem as we obtain $C_{\max }=C_{\max }^{2}$. If $S_{m}>I_{m}$, then $C_{\max }>I_{m}+p_{m}=C_{\max }^{2}$. In this case, we can use the optimal schedule for $(A 2)$ on $M_{1}, M_{2}, \ldots, M_{m-1}$ as a schedule for the original problem and start the continuous processing of the sublots at $t=S_{m}$ on $M_{m}$. The resulting schedule will have $C_{\max }=S_{m}+p_{m}$ and so it must be optimal

By the consecutive application of the above two lemmas, we obtain the following theorem.

Theorem 5 The $m$ machine problem is equivalent to an alternative problem (A3) in which there is no setup time on the first and last machine and the setup time on the ith machine is reduced to $S_{i}^{\prime}=\max \left\{S_{i}-S_{1}, 0\right\}$ for $i=2, . ., m-1$. If $C_{\max }^{3}$ is the optimal makespan for (A3), then the optimal sublot sizes for $(A 3)$ are also optimal for the original problem and $C_{\max }=\max \left\{C_{\max }^{3}, p_{m}+\max \left\{S_{m}-S_{1}, 0\right\}\right\}+S_{1}$.

From this theorem, we immediately get the following corollary.

Corollary 6 The three-machine problem with detached setup times $S_{1}, S_{2}$ and $S_{3}$ on $M_{1}, M_{2}$ and $M_{3}$, respectively, is equivalent to an alternative (reduced) problem with no setup time on $M_{1}$ and $M_{3}$ and setup time $S_{2}^{\prime}$ $=\max \left\{S_{2}-S_{1}, 0\right\}$ on $M_{2}$.

Motivated by the previous Corollary, we restrict our attention to a special case of problem $(A 3)$ in which $S_{i}^{\prime}=0$ for $i \neq 2$. We refer to this as the reduced problem. If we set $S_{2}^{\prime}=0$ too in a reduced problem, then this is a problem with no setup times, which we refer to as the relaxed problem.

In the remainder of this section, we study the structure of optimal solutions for the reduced problem, with the assumption that there is an optimal solution with consistent sublots. Following the approach in [13], such a solution can be represented by a network $N(x)$ which contains a vertex for each setup and each sublot on every machine (see Fig. 1).

In the network, $x_{0}$ is a dummy variable and $x_{i}, i=1,2, \ldots, s$, is the sublot size. The directed arc from vertex $(i, j)$ to vertex $(i+1, j)(i<m)$ represents the production constraint that sublot $j$ can be processed on machine $(i+1)$ 
only if it is completed on machine $i$. The directed arc from vertex $(i, j)$ to vertex $(i, j+1) \quad(j<s)$ represents the machine capacity constraint that sublot $(j+1)$ can start on $M_{i}$ only when the $j$ th sublot is completed on it. The vertex $(i, 0)$ has weight 0 for $i \neq 2$ and $S_{2}^{\prime}$ for $i=2$, and vertex $(i, j)$ has weight $p_{i} x_{j}$ for $1 \leq i \leq m, 1 \leq j \leq s$.

Using the network representation, the objective becomes to determine the sublot sizes which minimize the length of the longest path in the network, where the length of any path is the sum of the weights of the vertices on it. Any longest path is referred to as a critical path. A subpath of a (critical) path is called a (critical) segment. We call sublot $j$ critical in $N(x)$ if there is a critical segment containing the arc from $(i, j)$ to $(i+1, j)$ for some $i \in\{1,2, \ldots, m-1\}$.

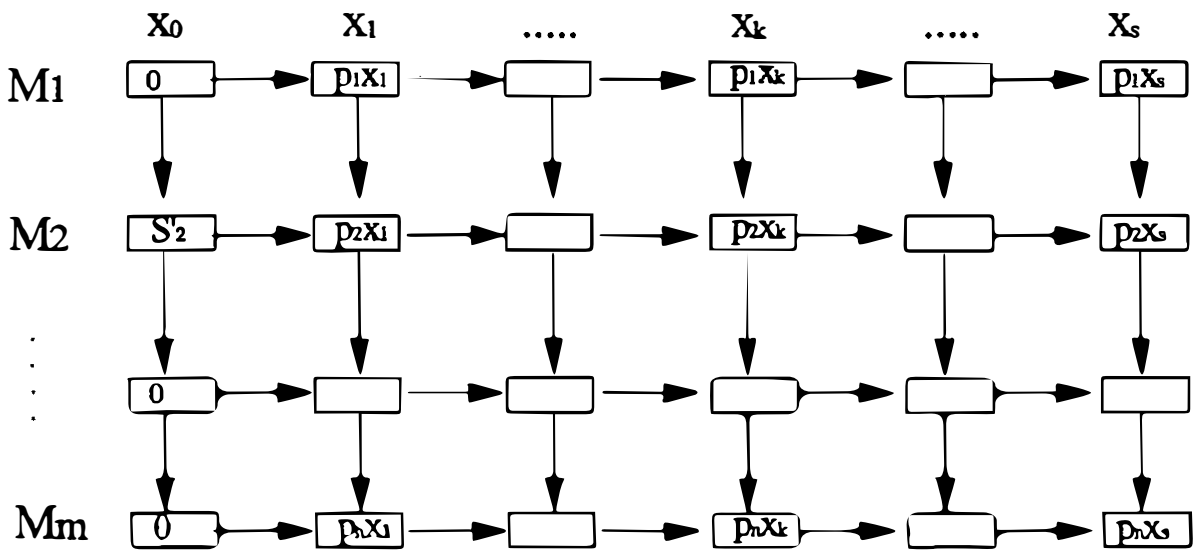

Figure 1: The network for a solution with consistent sublots

Theorem 7 Consider an optimal solution with consistent sublots for the reduced problem on $m$ machines, then every sublot $k$ is critical in the corresponding optimal network for $1 \leq k \leq s$. If $S_{2}^{\prime} \geq p_{1} x_{1}^{R}$, then sublot 0 is 
also critical, where $x_{1}^{R^{\prime}}$ is the optimal size of the first sublot for the relaxed problem.

Proof. For any vector $x=\left(x_{1}, x_{2}, \ldots, x_{s}\right)$ of the sublot sizes, let $L\left(i, j, i^{\prime}, j^{\prime}, x\right)$ represent the length of the longest path from vertex $(i, j)$ to vertex $\left(i^{\prime}, j^{\prime}\right)$ and let $M(x)$ be the length of the longest path from $(1,0)$ to $(m, s)$.

For any machine $h$ and $i(1 \leq h \leq i \leq m)$ and any sublot $j$, where $1 \leq j \leq s$, let $H(h, i, j, x)$ be the length of the longest path from $(1,0)$ to $(m, s)$ containing the vertical segment $(h, j)-\ldots-(i, j)$. Thus,

$H(h, i, j, x)=L(1,0, h, j-1, x)+x_{j} \sum_{g=h}^{i} p_{g}+L(i, j+1, m, s, x) \quad 1 \leq j \leq s$,

assuming $L(i, s+1, m, s, x)=0$. So, for any sublot $j$,

$$
M(x)=\max _{1 \leq h \leq i \leq m}\{H(h, i, j, x)\} .
$$

Now let $x=\left(x_{1}, x_{2}, \ldots, x_{s}\right)$ be a vector of optimal sublot sizes, which yields the minimum makespan $M(x)$, and suppose that sublot $k$ is noncritical. Because sublot $k$ is non-critical, each segment $(h, k)-\ldots-(i, k)$ $(h<i)$ is non-critical and we have

$$
M(x)=\max _{1 \leq i \leq m}\{H(i, i, k, x)\} .
$$

We construct $x^{\prime}=\left(x_{1}^{\prime}, x_{2}^{\prime}, \ldots, x_{s}^{\prime}\right)$, where $x_{j}^{\prime}=(1-\varepsilon) x_{j}$ for $1 \leq j \leq k-1$ and $k+1 \leq j \leq s$ and $x_{k}^{\prime}=x_{k}(1-\varepsilon)+\varepsilon$. From (1), $M\left(x^{\prime}\right)=H\left(h, i, k, x^{\prime}\right)$ for some $h$ and $i$, satisfying $h \leq i$. We show that $H\left(h, i, k, x^{\prime}\right)<M(x)$, contradicting the assumption that $x$ yields the minimum makespan. There are two cases.

Case 1. $\quad h<i$

$$
\text { Let } \quad \varepsilon=\left\{M(x)-\max _{1 \leq e<f \leq m}(H(e, f, k, x))\right\} /\left(\sum_{g=1}^{m} p_{g}+S_{2}^{\prime}\right)
$$

Since sublot $k$ is non-critical, $\varepsilon>0$, and note that $\varepsilon<1$. 
If $h=1$, then the longest path from $(1,0)$ to $(m, s)$ which contains the segment $(h, j)-, \ldots-(i, j)$ must avoid the setup node on $M_{2}$, and therefore,

$$
\begin{aligned}
H\left(h, i, k, x^{\prime}\right)= & (1-\varepsilon) L(1,0, h, k-1, x)+\left(x_{k}(1-\varepsilon)+\varepsilon\right) \sum_{g=1}^{i} p_{g}+ \\
& (1-\varepsilon) L(i, k+1, m, s, x) \\
= & (1-\varepsilon) H(h, i, k, x)+\varepsilon \sum_{g=1}^{i} p_{g} \\
< & H(h, i, k, x)+\varepsilon \sum_{g=1}^{m} p_{g} \\
< & M(x)
\end{aligned}
$$

where the last inequality follows from equation (2), which defines $\varepsilon$. If $h>1$ and the setup $S_{2}^{\prime}$ is not contained in $L\left(1,0, h, k-1, x^{\prime}\right)$, then

$$
\begin{aligned}
H\left(h, i, k, x^{\prime}\right)= & (1-\varepsilon) L(1,0, h, k-1, x)+\left(x_{k}(1-\varepsilon)+\varepsilon\right) \sum_{g=h}^{i} p_{g}+ \\
& (1-\varepsilon) L(i, k+1, m, s, x) .
\end{aligned}
$$

If $h>1$ and $S_{2}^{\prime}$ is contained in $L\left(1,0, h, k-1, x^{\prime}\right)$, then

$$
\begin{aligned}
H\left(h, i, k, x^{\prime}\right) \leq & (1-\varepsilon) L(1,0, h, k-1, x)+\left(x_{k}(1-\varepsilon)+\varepsilon\right) \sum_{g=h}^{i} p_{g}+ \\
& (1-\varepsilon) L(i, k+1, m, s, x)+\varepsilon S_{2}^{\prime} .
\end{aligned}
$$

Therefore, in either case, for $h>1$

$$
\begin{aligned}
H\left(h, i, k, x^{\prime}\right) \leq & (1-\varepsilon) L(1,0, h, k-1, x)+\left(x_{k}(1-\varepsilon)+\varepsilon\right) \sum_{g=h}^{i} p_{g}+ \\
& (1-\varepsilon) L(i, k+1, m, s, x)+\varepsilon S_{2}^{\prime} \\
& =(1-\varepsilon) H(h, i, k, x)+\varepsilon \sum_{g=h}^{i} p_{g}+\varepsilon S_{2}^{\prime} \\
& <H(h, i, k, x)+\varepsilon \sum_{g=h}^{m} p_{g}+\varepsilon S_{2}^{\prime} \\
& \leq M(x),
\end{aligned}
$$

where the last inequality follows from (2) again.

Case 2. $h=i$

Let $\varepsilon>0$ be arbitrary. 
If $h=i=1$, then

$$
\begin{aligned}
H\left(h, i, k, x^{\prime}\right)= & (1-\varepsilon) L(1,0, h, k-1, x)+\left(x_{k}(1-\varepsilon)+\varepsilon\right) \sum_{g=1}^{1} p_{g} \\
& \quad+(1-\varepsilon) L(i, k+1, m, s, x) \\
= & H(h, i, k, x)-\varepsilon\left(H(h, i, k, x)-p_{1}\right),
\end{aligned}
$$

Using $H(h, i, k, x)>p_{1}$ in (3), we get

$$
H\left(h, i, k, x^{\prime}\right)<H(h, i, k, x) \leq M(x) .
$$

If $h=i \geq 2$ and the setup $S_{2}^{\prime}$ is not contained in $L\left(1,0, h, k-1, x^{\prime}\right)$, then

$$
H\left(h, i, k, x^{\prime}\right)=(1-\varepsilon) L(1,0, h, k-1, x)+\left(x_{k}(1-\varepsilon)+\varepsilon\right) p_{i}+
$$

$$
(1-\varepsilon) L(i, k+1, m, s, x) \text {. }
$$

If $h=i \geq 2$ and the setup $S_{2}^{\prime}$ is contained in $L\left(1,0, h, k-1, x^{\prime}\right)$, then

$$
\begin{aligned}
H\left(h, i, k, x^{\prime}\right) \leq & (1-\varepsilon) L(1,0, h, k-1, x)+\left(x_{k}(1-\varepsilon)+\varepsilon\right) p_{i}+ \\
& (1-\varepsilon) L(i, k+1, m, s, x)+\varepsilon S_{2}^{\prime} .
\end{aligned}
$$

Therefore, in either case, if $h=i \geq 2$, then

$$
\begin{aligned}
H\left(h, i, k, x^{\prime}\right) \leq & (1-\varepsilon) L(1,0, h, k-1, x)+\left(x_{k}(1-\varepsilon)+\varepsilon\right) p_{i} \\
& +(1-\varepsilon) L(i, k+1, m, s, x)+\varepsilon S_{2}^{\prime} \\
= & (1-\varepsilon) H(i, i, k, x)+\varepsilon S_{2}^{\prime}+\varepsilon p_{i} \\
= & H(i, i, k, x)-\varepsilon\left(H(i, i, k, x)-S_{2}^{\prime}-p_{i}\right) .
\end{aligned}
$$

$H(i, i, k, x)$ should be longer than $S_{2}^{\prime}+p_{i}$, so from (4), we get again $H\left(h, i, k, x^{\prime}\right)<H(h, i, k, x) \leq M(x)$.

Thus, we have proved in every case that $M\left(x^{\prime}\right)<M(x)$, which contradicts the assumption that $x$ yields the minimum makespan. Therefore, sublot $k$ must be critical for $1 \leq k \leq s$.

Now we consider the dummy sublot 0 . We show that it is also critical if $S_{2}^{\prime} \geq p_{1} x_{1}^{R}$.

The relaxed problem has no setups. The structure of the optimal network for such a problem was described in detail in [6]. Of this, we need only the fact that every sublot is critical in an optimal solution of the relaxed problem. In particular, sublot 1 is critical, which implies that $(1,1)-(2,1)$ is always a critical segment. If we add the setup time $S_{2}^{\prime}$, we have to compare the lengths of the segments $(1,0)-(2,0)-(2,1)$ and $(1,0)-(1,1)-(2,1)$. 
The first segment has length $S_{2}^{\prime}+p_{2} x_{1}^{R}$ and the second segment has length $p_{1} x_{1}^{R}+p_{2} x_{1}^{R}$. If $S_{2}^{\prime} \leq p_{1} x_{1}^{R}$, then the setup does not affect the length of the critical path, thus it can be ignored and the reduced problem is equivalent to the relaxed problem. If $S_{2}^{\prime}>p_{1} x_{1}^{R}$, then the segment $(1,0)-(2,0)-(2,1)$ is always at least as long as $(1,0)-(1,1)-(2,1)$ in any optimal solution $x$ for the reduced problem, otherwise, $H(1,2,1, x)=M(x)$ and we could reduce $M(x)$ by reducing $x_{1}[6]$, contradicting the optimality of $x$.

The above proof indicates that the reduced problem is really different from the relaxed problem only if $S_{2}^{\prime}>p_{1} x_{1}^{R}$. For the remainder of the paper, we assume that this is the case we are dealing with, so that the segment $(1,0)-(2,0)$ is always critical.

Corollary 8 There is a consistent-sublot optimal solution for the reduced problem on three machines in which every sublot is critical.

Corollary 9 In any consistent sublot optimal solution of the reduced problem on $m$ machines, all the sublot sizes are positive.

Proof. Let $x$ be the optimal sublot vector and assume there are sublots $k$ and $j$ for which $x_{k}=x_{k+1}=\ldots=x_{j}=0, j \geq k$. There are two cases.

Case 1. $x_{j+1} \neq 0$

By Theorem 7, there exist $h$ and $i(1 \leq h<i \leq m)$ for which $(h, k), \ldots,(i, k)$ is a critical segment for the sublot $k$, i.e.,

$$
M(x)=H(h, i, k, x) .
$$

Since $x_{k}=x_{k+1}=\ldots=x_{j}=0$,

$$
H(h, i, k, x)=L(1,0, h, k-1, x)+L(i, j+1, m, s, x) .
$$

There is an alternative path, however, which contains the segment $(h, k)-(h, k+1)-\ldots-(h, j+1)-(h+1, j+1)-\ldots-(i, j+1)$ with length $L(1,0, h, k-1, x)+L(h, k, h, j, x)+L(h, j+1, i, j+1, x)+L(i, j+1, m, s, x)$. Using (5) and (6) and the fact that $L(h, j+1, i, j+1, x)>0$, we see that this alternative path is longer than $M(x)$, a contradiction.

Case $2: j=s$

According to Theorem 7 , there must be a critical segment for sublot $k$, say $(h, k)-(h+1, k)$. Let the critical path containing $(h, k)-(h+1, k)$ have 
the length $L E N 1$, then since $x_{k+1}=\ldots=x_{s}=0$,

$$
L E N 1=L(1,0, h, k-1, x) .
$$

There is an alternative path, however, which coincides with the above critical path until vertex $(h, k-1)$ and has the segment $(h, k-1)-(h+1, k-1)$. If its length is $L E N 2$, then

$$
L E N 2=L(1,0, h, k-1, x)+x_{k-1} p_{h+1}>L E N 1 .
$$

This is in contradiction with the assumption that $L E N 1$ is the length of a critical path.

Corollary 10 There is a consistent-sublot optimal solution for the reduced problem on three machines in which all sublot sizes are positive.

Observation 1 We call $(i, l)$ an upper critical corner in the network if there is a critical path containing $(i, k)-(i, k+1)-\ldots-(i, l)-(i+1, l)-\ldots-(g, l)$, where $1 \leq k<l$. An upper critical corner has the following property:

$$
\left(\sum_{j=k}^{l} x_{j}\right) p_{i}+\left(\sum_{j=i+1}^{g} p_{j}\right) x_{l} \geq\left(\sum_{j=i}^{g} p_{j}\right) x_{k}+\left(\sum_{j=k+1}^{l} x_{j}\right) p_{g} .
$$

Observation 2 We call $\left(i^{\prime}, k\right)$ a lower critical corner in the network if $k \geq 1$ and there is a critical segment $(i, k)-\ldots-\left(i^{\prime}, k\right)-\left(i^{\prime}, k+1\right)-\ldots-\left(i^{\prime}, l\right)$, where $i^{\prime}>i$. A lower critical corner $\left(i^{\prime}, k\right)$ has the following property :

$$
\left(\sum_{j=k}^{l} x_{j}\right) p_{i}+\left(\sum_{j=i+1}^{i^{\prime}} p_{j}\right) x_{l} \leq\left(\sum_{j=i}^{i^{\prime}} p_{j}\right) x_{k}+\left(\sum_{j=k+1}^{l} x_{j}\right) p_{i^{\prime}} .
$$

Observation 3 We call $(i, l)$ and $\left(i^{\prime}, k\right)$ matching critical corners in the network if $i<i^{\prime}, 1 \leq k<l$ and both $(i, k)-\ldots-(i, l)-(i+1, l)-\ldots-\left(i^{\prime}, l\right)$ and $(i, k)-\ldots-\left(i^{\prime}, k\right)-\ldots-\left(i^{\prime}, l\right)$ are critical segments. Matching critical corners $(i, l)$ and $\left(i^{\prime}, k\right)$ have the following property:

$$
\left(\sum_{j=k}^{l} x_{j}\right) p_{i}+\left(\sum_{j=i+1}^{i^{\prime}} p_{j}\right) x_{l}=\left(\sum_{j=i}^{i^{\prime}} p_{j}\right) x_{k}+\left(\sum_{j=k+1}^{l} x_{j}\right) p_{i^{\prime}}
$$

\section{Network Structure on Three Machines}

We first study the two-machine reduced problem with setup time $S_{2}^{\prime}$ on $M_{2}$.

Theorem 11 The optimal sublot sizes for the two-machine relaxed problem are also optimal for the reduced problem. 
Proof. Let $x_{1}^{r}$ be the optimal first sublot size in the relaxed problem on two machines.

If $S_{2}^{\prime} \leq p_{1} x_{1}^{r}$, it is obvious that adding the setup time $S_{2}^{\prime}$ to the relaxed problem will not affect the optimal solution.

If $S_{2}^{\prime}>p_{1} x_{1}^{r}$, by making the sublot sizes equal to the optimal sublot sizes in the relaxed problem, the critical path in the network is $(1,0)-(2,0)-$ $(2,1)-\ldots-(2, s)$. The makespan of this schedule is $S_{2}^{\prime}+p_{2}$. This is also the lower bound, however, for the optimal makespan. Therefore, the optimal sublot sizes for the relaxed problem are also optimal for the reduced problem on two machines.

From now on, we focus on the reduced three-machine problem. By the above analysis, we know that we only need to consider consistent sublots for the problem and every sublot is critical. Similarly to the relaxed problem [6], we have to distinguish three cases, depending on whether $\left(p_{2}\right)^{2}<p_{1} p_{3}$, $\left(p_{2}\right)^{2}=p_{1} p_{3}$ or $\left(p_{2}\right)^{2}>p_{1} p_{3}$.

Case $1 \quad\left(p_{2}\right)^{2}<p_{1} p_{3}$

If $S_{2}^{\prime}$ is "very large", it is clear that no horizontal segment on $M_{1}$ can be critical. Later we calculate the exact threshold value for $S_{2}$ (see Remark 1 after Corollary 18). If $S_{2}^{\prime}$ exceeds this value, then the problem becomes a relaxed two-machine problem (on $M_{2}$ and $M_{3}$ ), which was solved in constant time in [14]. In the meantime, we consider only the other case, in which there is a critical segment on $M_{1}$.

Lemma 12 If $\left(p_{2}\right)^{2}<p_{1} p_{3}$, then there is no critical segment of the form $(1, k)-(2, k)-\ldots-(2, l)-(3, l)$ with $l>k \geq 1$.

Proof. If $(1, k)-(2, k)-\ldots-(2, l)-(3, l)$ is a critical segment, then $(2, k)$ and $(2, l)$ must be lower and upper critical corners, respectively. Using Observation 2 for $(2, k)$, we obtain

$$
p_{1} x_{k}+p_{2} \sum_{j=k}^{l} x_{j} \geq p_{1} \sum_{j=k}^{l} x_{j}+p_{2} x_{l},
$$

which simplifies to

$$
p_{2} \sum_{j=k}^{l-1} x_{j} \geq p_{1} \sum_{j=k+1}^{l} x_{j}
$$


Using Observation 1 for $(2, l)$, we get

$$
p_{2} \sum_{j=k}^{l} x_{j}+p_{3} x_{l} \geq p_{2} x_{k}+p_{3} \sum_{j=k}^{l} x_{j}
$$

which simplifies to

$$
p_{2} \sum_{j=k+1}^{l} x_{j} \geq p_{3} \sum_{j=k}^{l-1} x_{j} .
$$

Multiplying (7) and (8) side by side yields a contradiction with the assumption $\left(p_{2}\right)^{2}<p_{1} p_{3}$.

The structure of the critical paths in this case is best described by Figure 2 , where heavy lines show critical segments, light lines non-critical segments and the dotted line could be critical or not depending on the actual data. We note that the critical path structure here is a lot more complex than in the case of no setups [6]. The following theorem summarizes the distinguishing properties of the critical paths.

Theorem 13 If $\left(p_{2}\right)^{2}<p_{1} p_{3}$, then there is a $k \epsilon\{1,2, \ldots, s\}$ such that

i) no segment $(1, j)-(2, j)$ is critical for $1 \leq j<k$;

ii) no segment $(2, j)-(2, j+1)$ is critical for $k \leq j<s$;

iii) the segment $(2, k-1)-(2, k)$ may or may not be critical, depending on the actual data;

iv) every other 2-node segment is critical, except $(2,0)-(3,0)$ and $(3,0)-$ $(3,1)$.

Proof. Suppose the $k$ th sublot is the first sublot that has critical segments $(1, k)-(2, k)-(3, k)$ in the optimal schedule. We can deduce that segments $(2, j)-(2, j+1)$ for $k \leq j \leq s-1$ are non-critical by Lemma 12 . By Corollary 8 , each sublot is critical, so at least one of the segments $(1, j)-(2, j),(2, j)-$ $(3, j)$ for $k \leq j \leq s$ is critical. Since $(2, j)-(2, j+1)$ cannot be critical for $k \leq j \leq s-1$, however, both segments $(1, j)-(2, j)$ and $(2, j)-(3, j)$ should be critical for $k \leq j \leq s$. 


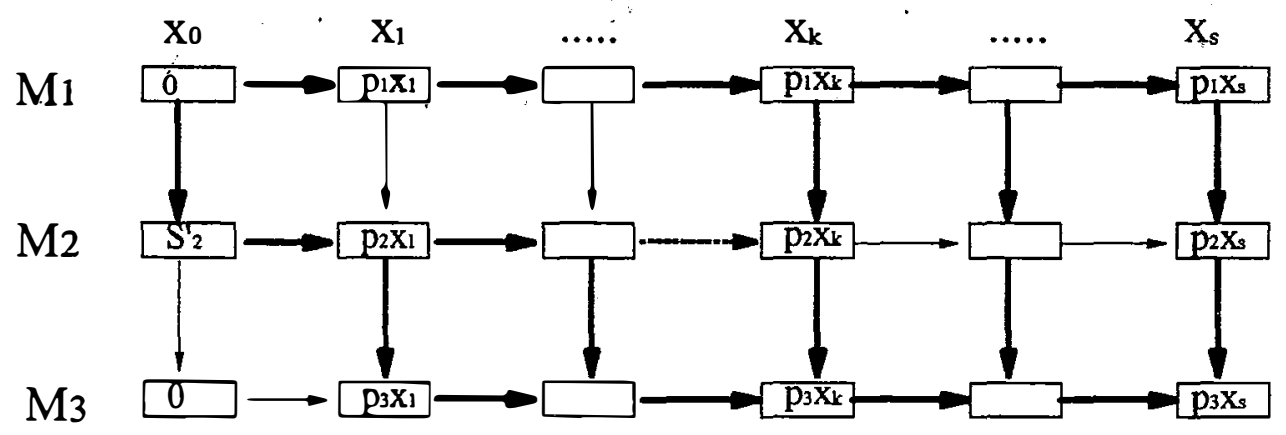

Figure 2: The network structure when $\left(p_{2}\right)^{2}<p_{1} p_{3}$

We show now that $(2, k-1)-(3, k-1)$ is critical. Suppose it is not, then by Corollary $8,(1, k-1)-(2, k-1)$ and $(2, k-1)-(2, k)$ must be critical segments, so $(2, k-1)$ and $(1, k)$ are matching critical corners. Together these imply :

$$
x_{k} p_{1}=x_{k-1} p_{2} \quad \text { and } \quad x_{k} p_{2}>x_{k-1} p_{3}
$$

which is in contradiction with our assumption of $\left(p_{2}\right)^{2}<p_{1} p_{3}$. So $(2, k-$ $1)-(3, k-1)$ must be a critical segment. Similar argument can be used to show that $(2, j)-(3, j)$ must be a critical segment for $1 \leq j \leq k-2$.

By definition, $\mathrm{k}$ is the first sublot which has critical segment $(1, k)-$ $(2, k)-(3, k)$, therefore, $(1, j)-(2, j)$ is not critical for $1 \leq j \leq k-1$.

Each critical segment $(2, j)-(3, j)$ for $1 \leq j \leq k-1$ must be part of a critical path, and since $(1, j)-(2, j)$ is non-critical, this implies that $(2, j-$ $1)-(2, j)$ must be critical. Finally, since $(2,0)-(2,1)$ is critical, $(1,0)-(2,0)$ must be critical too. The status of the dotted segment $(2, k-1)-(2, k)$ cannot be decided based on the above analysis. This will be demonstrated later by actual examples.

We note that when $S_{2}^{\prime}=0$, then $k=1$ and the optimal network structure reduces to the one proved in [6]. 


\section{Case $2 \quad\left(p_{2}\right)^{2}=p_{1} p_{3}$}

Theorem 14 If $\left(p_{2}\right)^{2}=p_{1} p_{3}$, the optimal network satisfies the following:

i) segment $(1,0)-(2,0)$ is critical;

ii) segments $(2, i)-(2, i+1),(2, j)-(3, j)$ and $(3, g)-(3, g+1)$ are critical for $0 \leq i<s, 1 \leq j \leq s$ and $1 \leq g<s$

iii) all other 2-node segments are non-critical.

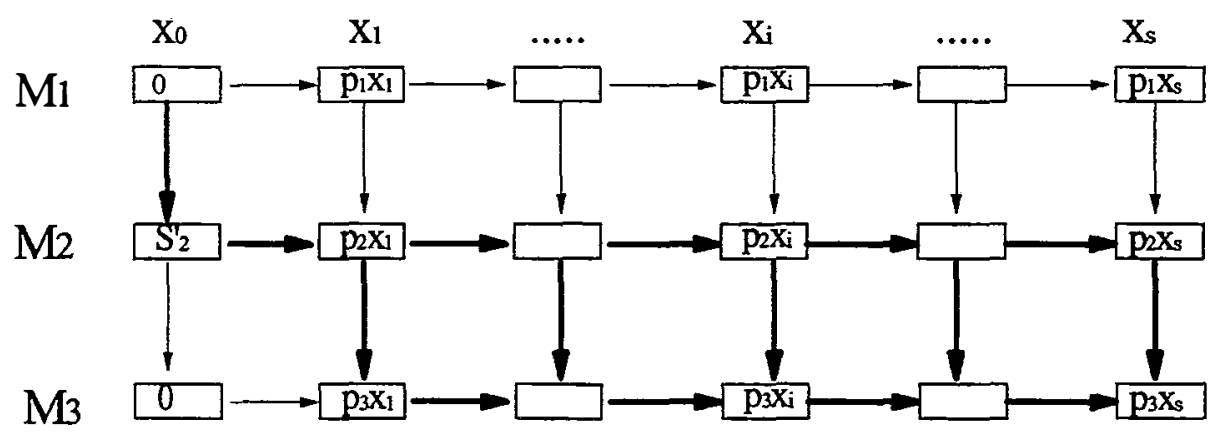

Figure 3: The network structure when $\left(p_{2}\right)^{2}=p_{1} p_{3}$

Proof. Suppose sublot $k$ is the last sublot which has a critical segment $(1, k)-(2, k)$. According to Theorem $7,(2, j)-(3, j)$ should be critical for $k<j \leq s$, so $(2, i)-(2, i+1)$ should also be critical for $k \leq i<s-1$, as otherwise we could not reach $(2, j)$ on a critical path. Thus $(2, k)$ and $(2, s)$ are lower and upper critical corners, respectively, unless $k=0$ or $k=s$. If $k \neq 0$ and $k \neq s$, then from Observations 1 and 2, we get

$$
p_{1} \sum_{i=k+1}^{s} x_{i}<p_{2} \sum_{i=k}^{s-1} x_{i} \quad \text { and } \quad p_{2} \sum_{i=k+1}^{s} x_{i} \geq p_{3} \sum_{i=k}^{s-1} x_{i} .
$$


The multiplication of these inequalities yields a contradiction, however, with the assumption of $\left(p_{2}\right)^{2}=p_{1} p_{3}$. Therefore, $k \notin\{1,2, \ldots, s-1\}$.

If $k=s$, then $(1, s)$ is an upper critical corner. Furthermore, we show that in this case the segment $(1, j)-(2, j)$ must be critical for $1 \leq j \leq s-1$. Suppose it was not for some $j \in\{1,2, \ldots, s-1\}$, then

$$
p_{2} \sum_{i=j}^{s-1} x_{i}<p_{1} \sum_{i=j+1}^{s} x_{i}
$$

By Theorem $7,(2, j)-(3, j)$ must be critical, implying that $(3, j)$ is a lower critical corner. By Observation 2,

$$
p_{3} \sum_{i=j}^{s-1} x_{i} \geq p_{2} \sum_{i=j+1}^{s} x_{i} .
$$

Multiplying (9) and (10), however, yields a contradiction with $\left(p_{2}\right)^{2}=p_{1} p_{3}$. Since $(1, s)-(2, s)$ is clearly critical, we have proved that if $k=s$, then $(1, j)-(2, j)$ is critical for $1 \leq j \leq s$.

We show now that segment $(2, s-1)-(2, s)$ is also critical, if $k=s$, and $(1, s)$ and $(2, s-1)$ must be matching critical corners, implying

$$
p_{2} x_{s-1}=p_{1} x_{s} .
$$

Suppose segment $(2, s-1)-(2, s)$ was not critical. Then segment $(1, s-$ 1) $-(2, s-1)-(3, s-1)-(3, s)$ must be critical, which means that $(1, s)$ and $(3, s-1)$ are matching critical corners, i.e.,

$$
\left(p_{2}+p_{3}\right) x_{s-1}=\left(p_{1}+p_{2}\right) x_{s} .
$$

But $\frac{p_{2}+p_{3}}{p_{1}+p_{2}}=\frac{p_{2}}{p_{1}}$ when $\left(p_{2}\right)^{2}=p_{1} p_{3}$, so (12) is equivalent to (11), contradicting the non-criticality of $(2, s-1)-(2, s)$. Thus, segments $(1, s-1)-(2, s-$ 1) $-(3, s-1)-(3, s)$ and $(2, s-1)-(2, s)$ must be critical if $k=s$.

Repeating this argument inductively for $i=s-1, s-2, \ldots, 1$, we can get

$$
p_{2} x_{i}=p_{1} x_{i+1} \text { for } 1 \leq i<s .
$$

Substituting (11) and (13) into $\sum_{i=1}^{s} x_{i}=1$, we obtain 


$$
x_{1}=\left\{\begin{array}{lll}
\frac{1-q_{1}}{1-q_{1}^{s}} & \text { if } & q_{1} \neq 1 \\
1 / s & \text { if } & q_{1}=1
\end{array} \text {, where } q_{1}=p_{2} / p_{1} .\right.
$$

This is the optimal first sublot size of the relaxed problem, i.e., $x_{1}=x_{1}^{R}$. Segment $(1,0)-(2,0)-(2,1)$ is critical, segment $(1,1)-(2,1)$ was shown to be critical above, so $S_{2}^{\prime}=p_{1} x_{1}^{R}$. This means that our problem is equivalent to the relaxed problem and we have excluded this case from our analysis by assuming that $S_{2}^{\prime}>p_{1} x_{1}^{R}$.

Therefore, $k \neq s$ and the only remaining possibility is $k=0$. The structure of the optimal network is shown in Fig. 3.

\section{Case $3 \quad\left(p_{2}\right)^{2}>p_{1} p_{3}$}

Similarly to Case 1, if $S_{2}^{\prime}$ is very large then no horizontal segment is critical on $M_{1}$. Later we calculate the exact threshold value for $S_{2}^{\prime}$ (see Remark 2 after Theorem 22). If $S_{2}^{\prime}$ exceeds this value, then this problem also becomes a relaxed two-machine problem (on $M_{2}$ and $M_{3}$ ), which was solved in [14]. In the meantime, we only consider the other case, when there is a critical segment on $M_{1}$.

Theorem 15 If $\left(p_{2}\right)^{2}>p_{1} p_{3}$, then there exist sublots $k$ and $j$, with $1 \leq k \leq$ $j \leq k+1$, such that

i) no segment $(1, i)-(2, i)$ is critical for $k<i \leq s$;

ii) no segment $(2, i)-(3, i)$, or $(3, i)-(3, i+1)$ is critical for $0 \leq i<j$;

iii) the segments $(2, k)-(3, k)$ and $(3, k)-(3, k+1)$ may or may not be critical, depending on the actual data;

iv) every other 2-node segment is critical.

Proof. Suppose $k$ is the last sublot with critical segment $(1, k)-(2, k)$ and $j$ is the first sublot with critical segment $(2, j)-(3, j)$. We show that $j \geq k$.

One extreme case is when $k=1$. In this case, it is obvious that $(2,0)-$ $(3,0)-(3,1)$ is not a critical segment and both $(1,0)-(2,0)-(2,1)$ and $(1,0)-(1,1)-(2,1)$ are critical segments. If $k>1$, then suppose $j<k$. This implies that $(1,0)-\ldots-(1, k)-(2, k)$ is a critical segment and $(2, j)-$ 


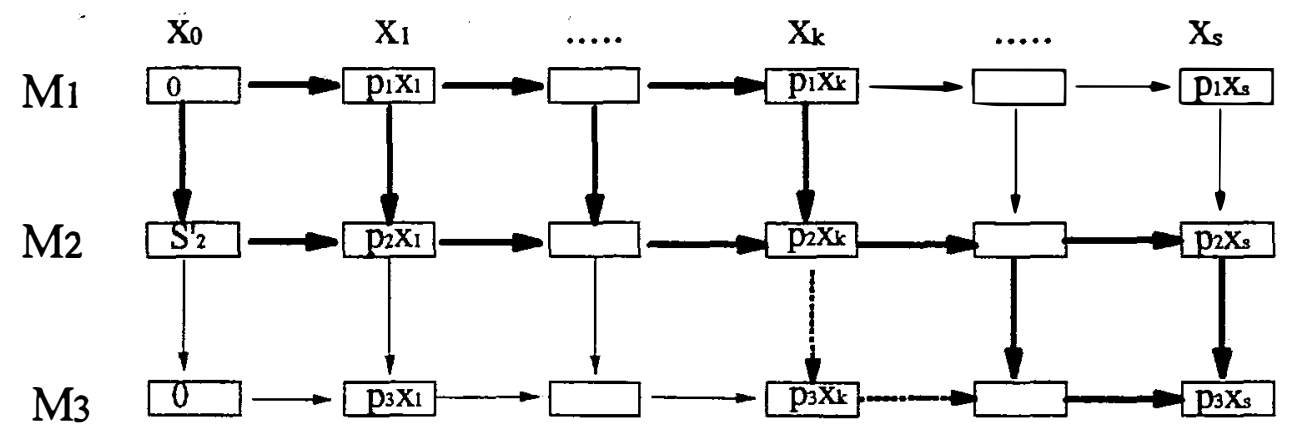

Figure 4: The network structure when $\left(p_{2}\right)^{2}>p_{1} p_{3}$

$(3, j)-\ldots-(3, k)$ is another critical segment. $(1, k)$ is an upper critical corner and $(3, j)$ is a lower critical corner, so by Observations 1 and 2 , we get

$$
p_{1} \sum_{i=j+1}^{k} x_{i} \geq p_{2} \sum_{i=j}^{k-1} x_{i} \quad \text { and } p_{3} \sum_{i=j}^{k-1} x_{i} \geq p_{2} \sum_{i=j+1}^{k} x_{i},
$$

which is in contradiction with the assumption $\left(p_{2}\right)^{2}>p_{1} p_{3}$. So $j \geq k$ indeed.

Finally, we cannot have $j>k+1$, as this would make sublot $k+1$ noncritical, contradicting Theorem 7 . We note that $j \geq k$ and Theorem 7 imply that $(1, i)-(2, i)$ must be critical for $i \epsilon\{1,2, \ldots, k\}$. The resulting network structure is depicted in Fig $4 . \square$

We will refer to sublot $k$ of Theorem 13 and 15 as the pattern-changing sublot.

\section{Optimal Sublot Sizes on Three Machines}

By knowing the characteristics of the critical paths in the network, we can find the optimal solution in each case. 
Case $1 \quad\left(p_{2}\right)^{2}<p_{1} p_{3}$,

Theorem 16 If $\left(p_{2}\right)^{2}<p_{1} p_{3}$ and $k$ is the index of the pattern-changing sublot, then the optimal sublot sizes are determined by

$$
\begin{array}{lll}
x_{1}=\frac{D-S_{2}^{\prime} B}{A D-B C}, & x_{j}=x_{1} q_{2}^{j-1} & \text { for } 1<j<k \\
x_{k}=\frac{C-S_{2}^{\prime} A}{B C-D A}, \quad x_{i}=x_{k} q^{i-k} & \text { for } k<i \leq s,
\end{array}
$$

where $A=\sum_{j=1}^{k-1} q_{2}^{j-1}, B=\sum_{i=1}^{s-k+1} q^{i-1}, C=\left(p_{1}-p_{3}\right) \sum_{j=1}^{k-1} q_{2}^{j-1}-p_{2}, D=p_{1}+p_{2}$, $q=\frac{p_{2}+p_{3}}{p_{1}+p_{2}}$ and $q_{2}=p_{3} / p_{2}$.

The optimal makespan is given by

$$
M(x)=x_{1} p_{1} \sum_{j=1}^{k-1} q_{2}^{j-1}+x_{k}\left(p_{1}+p_{2}+p_{3} \sum_{i=k}^{s} q^{i-k}\right) .
$$

Proof. According to Theorem $13,(3, j)$ and $(2, j+1)$ are matching critical corners for $1 \leq j \leq k-2$, so $p_{3} x_{j}=p_{2} x_{j+1}$ by Observation 3 . Therefore, $x_{j+1} / x_{j}=p_{3} / p_{2}=q_{2}$ for $1 \leq j \leq k-2$, or

$$
x_{j}=x_{1} q_{2}^{j-1} \quad \text { for } 1 \leq j \leq k-1
$$

Furthermore, $(3, i)$ and $(1, i+1)$ are also matching critical corners for $k \leq$ $i \leq s-1$, so $x_{i}\left(p_{2}+p_{3}\right)=x_{i+1}\left(p_{1}+p_{2}\right)$. Therefore, $x_{i+1} / x_{i}=\left(p_{2}+p_{3}\right) /\left(p_{1}+\right.$ $\left.p_{2}\right)=q$ for $k \leq i \leq s-1$, or

$$
x_{i}=x_{k} q^{i-k} \quad \text { for } k \leq i \leq s .
$$

Using $\sum_{j=1}^{s} x_{j}=1$ and the fact that $(1,0)-(1,1)-\ldots-(1, k)-(2, k)-(3, k)$ and $(1,0)-(2,0)-(2,1)-(3,1)-\ldots-(3, k)$ are critical segments, we get the following equations after substituting (16) and (17):

$$
\begin{gathered}
x_{1} \sum_{j=1}^{k-1} q_{2}^{j-1}+x_{k} \sum_{j=1}^{s-k+1} q^{j-1}=1 . \\
p_{1} x_{1} \sum_{j=1}^{k-1} q_{2}^{j-1}+x_{k}\left(p_{1}+p_{2}\right)=S_{2}^{\prime}+x_{1} p_{2}+x_{1} p_{3} \sum_{j=1}^{k-1} q_{2}^{j-1} .
\end{gathered}
$$


(18) and (19) can be rewritten as

$$
\begin{aligned}
& A x_{1}+B x_{k}=1 \\
& C x_{1}+D x_{k}=S_{2}^{\prime} .
\end{aligned}
$$

So, $x_{1}=\frac{D-S_{2}^{\prime} B}{A D-B C} ; \quad x_{k}=\frac{C-S_{2}^{\prime} A}{B C-A D}$.

Substituting (16) and (17) into the critical path $(1,0)-\ldots-(1, k)-$ $(2, k)-(3, k)-\ldots-(3, s)$, we obtain

$$
M(x)=x_{1} p_{1} \sum_{j=1}^{k-1} q_{2}^{j-1}+x_{k}\left(p_{1+} p_{2}\right)+x_{k} p_{3} \sum_{i=k}^{s} q^{i-k}
$$

In order to determine the actual value of $k$ which defines the patternchanging sublot, we show that varying the setup time $S_{2}^{\prime}$, while keeping all the other data (the processing times and $s$ ) fixed, will result in monotone changes in the value of $k$. Let $S_{2}(k)$ denote a setup time on $M_{2}$, for which $k$ is the pattern-changing sublot in the optimal solution for fixed $p_{i}(i=1,2,3)$ and $s$.

Theorem 17 If $\left(p_{2}\right)^{2}<p_{1} p_{3}$, then $k$ can increase only if $S_{2}(k)$ is increased sufficiently.

Proof. Let us denote the optimal sublot sizes by $x_{j}(k)(j=1,2, \ldots, s)$ if $k$ is the pattern-changing sublot. Note that $x_{j}(k)(j=1,2, \ldots, s)$ must satisfy the formulae of Theorem 13. Substituting $x_{j}(k)$ into (18), we obtain

$$
x_{1}(k)\left(1+q_{2}+\ldots+q_{2}^{k-2}\right)+x_{k}(k)\left(1+q+\ldots+q^{s-k}\right)=1 .
$$

$(3, k-1)$ is a lower and $(1, k)$ is an upper critical corner, so we get $x_{k-1}(k) p_{3} \geq$ $x_{k}(k) p_{2}$ and $x_{k}(k)\left(p_{1}+p_{2}\right) \geq x_{k-1}(k)\left(p_{2}+p_{3}\right)$, implying

$$
x_{k-1}(k) q \leq x_{k}(k) \leq x_{k-1}(k) q_{2} .
$$

Therefore, we have

$$
x_{1}(k)\left[\left(1+q_{2}+\ldots+q_{2}^{k-2}\right)+q_{2}^{k-1}\left(1+q+\ldots+q^{s-k}\right)\right] \geq 1
$$

Now we show that, for a fixed $x_{1}(k), k$ is the smallest positive integer number to satisfy inequality (22). For any integer number $k_{1}>k$, it is 
obvious that the inequality is valid with a fixed $x_{1}(k)$ because $q_{2}>q$. For any integer $k_{2}<k$, we obtain from (20) using (21)

$x_{1}(k)\left[\left(1+q_{2}+\ldots+q_{2}^{k_{2}-2}\right)+q_{2}^{k_{2}-1}\left(1+q_{2}+\ldots+q_{2}^{k-k_{2}-1}\right)\right]+x_{k-1}(k) q\left(1+q+\ldots+q^{s-k}\right) \leq 1$.

Using $x_{k-1}(k)=x_{1}(k) q_{2}^{k-2}$ and $q_{2}>q$, we get

$x_{1}(k)\left[\left(1+q_{2}+\ldots+q_{2}^{k_{2}-2}\right)+q_{2}^{k_{2}-1}\left(1+q_{2}+\ldots+q_{2}^{k-k_{2}-1}\right)+q_{2}^{k-2} q\left(1+q+\ldots+q^{s-k}\right)\right]<1$,

implying

$$
x_{1}(k)\left[\left(1+q_{2}+\ldots+q_{2}^{k_{2}-2}\right)+q_{2}^{k_{2}-1}\left(1+q+\ldots+q^{s-k_{2}}\right)\right]<1 .
$$

Thus, with a fixed $x_{1}(k), k$ is indeed the smallest positive integer to satisfy the inequality (22) .

Let us study now the relationship between $x_{1}(k)$ and $k$. Consider two problems where $k$ and $k_{1}\left(k<k_{1}\right)$ are the pattern-changing sublots, respectively. If we had $x_{1}(k) \leq x_{1}\left(k_{1}\right)$, then replacing $x_{1}(k)$ in (22) by $x_{1}\left(k_{1}\right)$ yields

$$
x_{1}\left(k_{1}\right)\left(\left(1+q_{2}+\ldots+q_{2}^{k-2}\right)+q_{2}^{k-1}\left(1+q+\ldots+q^{s-k}\right)\right) \geq 1 .
$$

This contradicts, however, the fact that $k_{1}$ is the smallest positive integer for which $x_{1}\left(k_{1}\right)$ and $k_{1}$ satisfy (22). Thus we must have $x_{1}(k)>x_{1}\left(k_{1}\right)$ if $k<k_{1}$.

Now we show that $x_{s}(k+1)>x_{s}(k)$. From (15)

$$
\frac{x_{j}(k)}{x_{s}(k)}=\left(\frac{1}{q}\right)^{s-j} \text { for } k<j \leq s \text { and } \frac{x_{j}(k+1)}{x_{s}(k+1)}=\left(\frac{1}{q}\right)^{s-j} \text { for } k+1<j \leq s .
$$

Therefore, we have

$$
\frac{x_{j}(k)}{x_{s}(k)}=\frac{x_{j}(k+1)}{x_{s}(k+1)} \quad \text { for } k+1<j \leq s .
$$

$(1, k+1)$ and $(3, k)$ are matching critical corners if the pattern-changing sublot is $k$ and $(1, k+1)$ is an upper critical corner if the pattern-changing sublot changes to $k+1$, therefore $x_{k}(k)\left(p_{2}+p_{3}\right)=x_{k+1}(k)\left(p_{1}+p_{2}\right)$, and $x_{k}(k+$ 1) $\left(p_{2}+p_{3}\right)<x_{k+1}(k+1)\left(p_{1}+p_{2}\right)$, implying 


$$
\frac{x_{k}(k+1)}{x_{k+1}(k+1)}<\frac{x_{k}(k)}{x_{k+1}(k)}
$$

Multiplying both sides of $(24)$ by $1 /\left(q^{s-k-1}\right)$, we get

$$
\frac{x_{k}(k+1)}{x_{s}(k+1)}<\frac{x_{k}(k)}{x_{s}(k)}
$$

From (14), we have

$$
\frac{x_{i}(k+1)}{x_{k-1}(k+1)}=\frac{x_{i}(k)}{x_{k-1}(k)}=\left(\frac{1}{q_{2}}\right)^{k-i-1} \quad \text { for } 1 \leq i \leq k-1 .
$$

Because $(3, k-1)$ is a lower critical corner if the pattern-changing sublot is $k$, and $(3, k-1)$ and $(2, k)$ are matching critical corners if the pattern-changing sublot is $k+1$, we have $x_{k-1}(k) p_{3} \geq x_{k}(k) p_{2}$ and $x_{k-1}(k+1) p_{3}=x_{k}(k+1)$ $p_{2}$, implying

$$
\frac{x_{k-1}(k+1)}{x_{k}(k+1)} \leq \frac{x_{k-1}(k)}{x_{k}(k)} .
$$

Multiplying (25) with (26) and (27), we obtain

$$
\frac{x_{i}(k+1)}{x_{s}(k+1)}<\frac{x_{i}(k)}{x_{s}(k)} \quad \text { for } \quad 1 \leq i \leq k-1 \text {. }
$$

Summing (23), (25), (28) and using the fact that $\sum_{i=1}^{s} x_{i}(k)=\sum_{i=1}^{s} x_{i}(k+1)=1$, we get

$$
\frac{1}{x_{s}(k+1)}<\frac{1}{x_{s}(k)}
$$

Therefore, $x_{s}(k+1)>x_{s}(k)$ indeed.

Consider now the optimal network when $k$ is the pattern-changing sublot. The paths $(1,0)-(1,1)-\ldots-(1, s)-(2, s)-(3, s)$ and $(1,0)-(2,0)-$ $(2,1)-(3,1)-\ldots-(3, s)$ are both critical, so they should have the same lengths, i.e.,

$$
p_{1}+\left(p_{2}+p_{3}\right) x_{s}(k)=S_{2}(k)+p_{2} x_{1}(k)+p_{3} .
$$

We have proved that when $k$ increases, $x_{1}(k)$ decreases and $x_{s}(k)$ increases. Therefore, since (29) must hold, $k$ can increase only if $S_{2}(k)$ increased. 
Corollary 18 When $\left(p_{2}\right)^{2}<p_{1} p_{3}$, the value of $k$ can be found in $O(\log s)$ time.

Proof. Let $S_{\max }(k)$ be the maximum value of $S_{2}^{\prime}$ at which $k$ is the patternchanging sublot. We show that $(2, k-1)-(2, k)$ is critical if $S_{2}^{\prime}=S_{\max }(k)$.

Suppose it was not, then $x_{k}(k)<x_{k-1}(k) q_{2}=x_{1}(k) q_{2}^{k}$. Substituting this into $(20)$, we obtain

$$
x_{1}(k)\left(\left(1+q_{2}+\ldots+q_{2}^{k-2}\right)+q_{2}^{k-1}\left(1+q+\ldots+q^{s-k}\right)\right)>1 .
$$

Now if we increase $S_{2}^{\prime}$ slightly, then $x_{1}(k)$ changes only slightly by Theorem 16 , so the inequality still holds for $k$, which implies that $k$ is still the patternchanging sublot. This, however, contradicts the assumption of $S_{2}^{\prime}=S_{\max }(k)$.

Therefore, we can find $k$ by binary search in the interval $[1, s]$. Start with the trial value $k_{0}=\lceil s / 2\rceil$. Using the fact that $\left(2, k_{0}-1\right)-\left(2, k_{0}\right)$ must be critical, (20) becomes

$$
x_{1}\left(k_{0}\right)\left(\left(1+q_{2}+\ldots+q_{2}^{k_{0}-2}\right)+q_{2}^{k_{0}-1}\left(1+q+\ldots+q^{s-k_{0}}\right)\right)=1,
$$

By the fact that both $(1,0)-(1,1)-\ldots-\left(1, k_{0}\right)-\left(2, k_{0}\right)$ and $(1,0)-(2,0)-$ $(2,1)-\ldots,-\left(2, k_{0}\right)$ must be critical segments, we have

$$
S_{\max }\left(k_{0}\right)+x_{1}\left(k_{0}\right) p_{2}\left(1+q_{2}+\ldots+q_{2}^{k_{0}-2}\right)=x_{1}\left(k_{0}\right) p_{1}\left(1+q_{2}+\ldots+q_{2}^{k_{0}-1}\right) .
$$

$S_{\max }\left(k_{0}\right)$ can be determined from (31) and (32) in $O(1)$ time. If $S_{\max }\left(k_{0}\right)<$ $S_{2}^{\prime}$, then $k_{0}<k$. Otherwise, $k_{0} \geq k$. Therefore, the binary search will end in $O(\log s)$ iterations.

Remark 1 The problem becomes a relaxed problem, if $S_{2}^{\prime}$ is greater than $S_{\max }(s)$. When $S_{2}^{\prime}=S_{\max }(s),(2, s-1)-(2, s)$ should be critical. Using Theorem 16 and the fact that $(1,0)-\ldots-(1, s)-(2, s)$ and $(1,0)-(2,0)-(2,1)-$ $\ldots-(2, s)$ are critical segments, we can get $S_{\max }(s)=p_{1}-p_{2} \frac{1-\left(p_{3} / p_{2}\right)^{s-1}}{1-\left(p_{3} / p_{2}\right)^{s}}$. Therefore, the setup time which makes the problem relaxed must satisfy $S_{2}^{\prime}>p_{1}-p_{2} \frac{1-\left(p_{3} / p_{2}\right)^{s-1}}{1-\left(p_{3} / p_{2}\right)^{s}}$.

The following example demonstrates how the presence of setups changes the optimal solution. It also shows that the dotted segment $(2, k-1)-(2, k)$ of Theorem 13 and Fig. 2 may or may not be critical, depending on the actual data. 

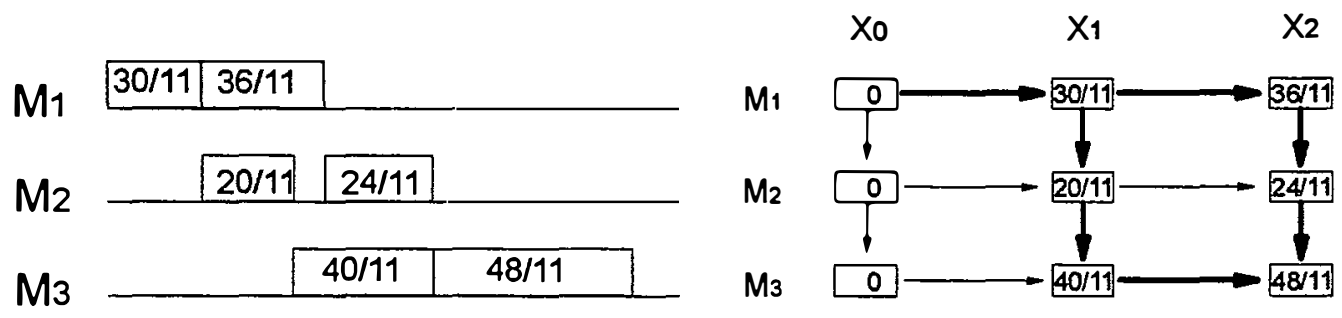

Figure 5: Case a. of Example 1

Example 1 Let $p_{1}=6 ; p_{2}=4 ; p_{3}=8 ; s=2$.

Case a. Let $S_{2}^{\prime}=0$.

In this case, $q=\frac{p_{2}+p_{3}}{p_{1}+p_{2}}=\frac{6}{5}$ and $k=1$. The optimal solution is $x_{1}=\frac{5}{11}$, $x_{2}=\frac{6}{11}$ and $M(x)=\frac{138}{11}$. It is clear that segment $(2,0)-(2,1)$ is not critical, but if we increased $S_{2}^{\prime}$ to $\frac{30}{11}$, then it would become critical.

Case b. Let $S_{2}^{\prime}=3$.
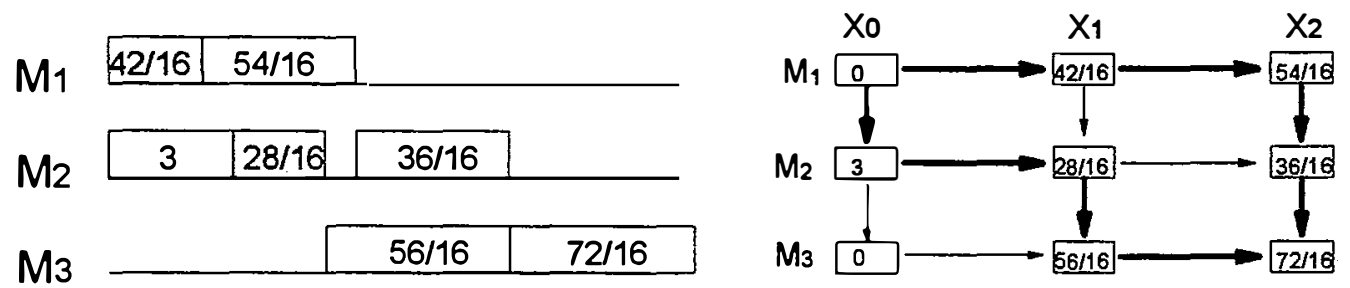

Figure 6: Case b. of Example 1

Since $S_{2}^{\prime}=3>\frac{30}{11}$, the solution obtained for Case a. is no longer optimal and sublot 2 becomes the pattern-changing sublot. In this case, 
$x_{1}=\frac{7}{16}, x_{2}=\frac{9}{16}$ and $M(x)=\frac{204}{16}$. The length of the longest path containing $(2,1)-(2,2)$ is $\frac{184}{16}$. Therefore, segment $(2,1)-(2,2)$ is not critical.

Case c. Let $S_{2}^{\prime}=\frac{14}{3}$.
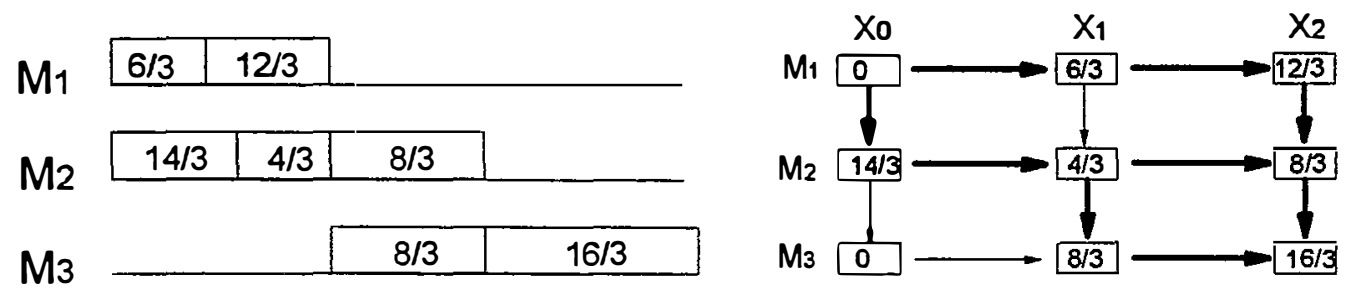

Figure 7: Case c. of Example 1

Using Theorem 16, we obtain $q_{2}=\frac{p_{3}}{p_{2}}=2, x_{1}=\frac{1}{3} ; x_{2}=\frac{2}{3}$ and $M(x)=$ 14. As it can be seen from Fig. $7,(2,1)-(2,2)$ is critical in this case. If we increased $S_{2}^{\prime}$ to more than $14 / 3$, then no horizontal segment on $M_{1}$ would be critical and the problem would become equivalent to a 2-machine problem on $M_{2}$ and $M_{3}$.

\section{Case $2 \quad\left(p_{2}\right)^{2}=p_{1} p_{3}$}

Theorem 19 When $\left(p_{2}\right)^{2}=p_{1} p_{3}$, the optimal sublot sizes are determined by $x_{1}=\left\{\begin{array}{cc}(1-q) /\left(1-q^{s}\right) & \text { if } p_{2} \neq p_{3} \\ 1 / s & \text { if } p_{2}=p_{3}\end{array} \quad x_{j}=q^{j-1} x_{1}, 1 \leq j \leq s\right.$,

where $q=\left(p_{2}+p_{3}\right) /\left(p_{1}+p_{2}\right)=p_{3} / p_{2}$.

The optimal makespan is

$M(x)=\left\{\begin{array}{lr}S_{2}^{\prime}+p_{2}(1-q) /\left(1-q^{s}\right)+p_{3} & \text { if } p_{2} \neq p_{3} \\ S_{2}^{\prime}+p_{2} / s+p_{3} & \text { if } p_{2}=p_{3}\end{array}\right.$

Proof. By Theorem $14,(2, j+1)$ and $(3, j)$ are matching critical corners for $1 \leq j \leq s-1$, so $x_{j} p_{3}=x_{j+1} p_{2}$, i.e., $x_{j+1} / x_{j}=p_{3} / p_{2}=q$. 
Substituting into $\sum_{i=1}^{s} x_{i}=1$, we obtain $x_{1}=(1-q) /\left(1-q^{s}\right)$ and $M(x)=$ $S_{2}^{\prime}+p_{2}(1-q) /\left(1-q^{s}\right)+p_{3}$ if $q \neq 1$ and $x_{1}=1 / s$ with $M(x)=S_{2}^{\prime}+p_{2} / s+p_{3}$ if $q_{2}=1$.

\section{Case $3 \quad\left(p_{2}\right)^{2}>p_{1} p_{3}$}

Theorem 20 For the case $\left(p_{2}\right)^{2}>p_{1} p_{3}$, the optimal sublot sizes satisfy

$$
\begin{aligned}
& x_{1}=S_{2}^{\prime} / p_{1}, \quad x_{i}=x_{1} q_{1}^{i-1} \quad \text { for } \quad 1 \leq i \leq k, \\
& x_{k+1}=\left\{\begin{array}{c}
\frac{\left(1-q_{1}\right) p_{1}-s_{2}^{\prime}\left(1-q_{1}^{k}\right)}{p_{1}\left(1-q_{2}^{s-k}\right)} \frac{1-q_{2}}{1-q_{1}} \quad \text { if } q_{1} \neq 1 \text { and } q_{2} \neq 1 \\
\left(p_{1}-S_{2}^{\prime} \sum_{i=0}^{k-1} q_{1}^{i}\right) /\left(p_{1} \sum_{i=0}^{s-k-1} q_{2}^{i}\right) \quad \text { otherwise }
\end{array}\right. \\
& x_{i}=x_{k+1} q_{2}^{i-k-1} \text { for } k+1 \leq i \leq s,
\end{aligned}
$$

where $q_{1}=p_{2} / p_{1}, q_{2}=p_{3} / p_{2}$, and $k$ is the pattern-changing sublot.

The optimal makespan is given by

$M(x)=S_{2}^{\prime} \sum_{i=0}^{k-1} q_{1}^{i}+S_{2}^{\prime} p_{2} q_{1}^{k-1} / p_{1}+x_{k+1}\left(p_{2}+p_{3} \sum_{i=0}^{s-k-1} q_{2}^{i}\right)$

Proof. According to Theorem 15, $(2, i)$ and $(1, i+1)$ are matching critical corners for $1 \leq i \leq k-1$, so $p_{1} x_{i+1}=p_{2} x_{i}$, implying

$$
x_{i}=x_{1} q_{1}^{i-1} \quad \text { for } 1 \leq i \leq k-1 .
$$

We know that $(2, i+1)$ and $(3, i)$ are also matching critical corners for $k+1 \leq$ $i \leq s-1$, so $p_{2} x_{i+1}=p_{3} x_{i}$, implying

$$
x_{i}=x_{k+1} q_{2}^{i-k-1} \quad \text { for } k+1 \leq i \leq s-1,
$$

Substituting (33) and (34) into $\sum_{i=1}^{s} x_{i}=1$, we get

$$
x_{1}\left(1+q_{1}+\ldots .+q_{1}^{k-1}\right)+x_{k+1}\left(1+q_{2}+\ldots+q_{2}^{s-k-1}\right)=1 .
$$

$\operatorname{Both}(1,0)-(1,1)-(2,1)$ and $(1,0)-(2,0)-(2,1)$ are critical, therefore

$$
x_{1} p_{1}=S_{2}^{\prime}
$$

Solving the above two equations, we obtain 


$$
x_{1}=S_{2}^{\prime} / p_{1}, \quad x_{k+1}=\left\{\begin{array}{l}
\frac{\left(1-q_{1}\right) p_{1}-S_{2}^{\prime}\left(1-q_{1}^{k}\right)}{p_{1}\left(1-q_{2}^{s-k}\right)} \frac{1-q_{2}}{1-q_{1}} \text { if } q_{1} \neq 1 \text { and } q_{2} \neq 1 \\
\left(p_{1}-S_{2}^{\prime} \sum_{i=0}^{k-1} q_{1}^{i}\right) /\left(p_{1} \sum_{i=0}^{s-k-1} q_{2}^{i}\right) \text { otherwise. }
\end{array}\right.
$$

Substituting into the makespan,

$$
M(x)=p_{1} \sum_{i=1}^{k} x_{i}+x_{k} p_{2}+x_{k+1} p_{2}+p_{3} \sum_{i=k+1}^{s} x_{i},
$$

which, after some easy algebraic manipulations, yields the formula in the theorem.

In order to determine the actual value of $k$ in this case, we show that varying the setup time $S_{2}^{\prime}$, while keeping all the other data (the processing times and $s$ ) fixed, will result in monotone changes in the value of $k$. Let $S_{2}(k)$ be a setup time on $M_{2}$ for which $k$ is the pattern-changing sublot in the optimal solution for fixed $p_{i}(i=1,2,3)$ and $s$.

Theorem 21 If $\left(p_{2}\right)^{2}>p_{1} p_{3}, k$ can increase only if $S_{2}(k)$ is decreased.

Proof. Let us denote the optimal sublot sizes by $x_{i}(k)(i=1,2, \ldots, s)$ if $k$ is the pattern-changing sublot. The $x_{i}(k)$ must satisfy the formulae of Theorem 20. Substituting $x_{i}(k)$ into (35), we get

$$
x_{1}(k)\left(1+q_{1}+\ldots+q_{1}^{k-1}\right)+x_{k+1}(k)\left(1+q_{2}+\ldots+q_{2}^{s-k-1}\right)=1
$$

By Theorem $15,(2, k)$ is a lower critical corner and $(1, k)-(1, k+1)$ is not critical, so $p_{1} x_{k+1}(k)<p_{2} x_{k}(k)$, implying

$$
x_{k+1}(k)<x_{k}(k) q_{1}=x_{1}(k) q_{1}^{k}=\left(S_{2}(k) / p_{1}\right) q_{1}^{k} .
$$

Therefore, if we replace $x_{k+1}(k)$ in (37), we get

$$
\left(S_{2}(k) / p_{1}\right)\left(1+q_{1}+\ldots+q_{1}^{k-1}+q_{1}^{k}\left(1+q_{2}+q_{2}^{2}+\ldots+q_{2}^{s-k-1}\right)\right)>1 .
$$

We show that $k$ is the minimum positive integer for which the inequality is valid for fixed $S_{2}(k)$. For any integer $k_{1}>k$, it is clear that the inequality is valid, because $q_{1}>q_{2}$. $(2, k+1)$ is an upper critical corner, so $x_{k+1}(k) p_{2} \geq$ $x_{k}(k) p_{3}$, implying

$$
x_{k+1}(k) \geq x_{k}(k) q_{2}=\left(S_{2}(k) / p_{1}\right) q_{1}^{k-1} q_{2} .
$$

Substituting this into (37), we obtain 
$1=\sum_{i=1}^{s} x_{i}(k) \geq\left(S_{2}(k) / p_{1}\right)\left(\left(1+q_{1}+\ldots+q_{1}^{k-1}\right)+q_{1}^{k-1} q_{2}\left(1+q_{2}+\ldots+q_{2}^{s-k-1}\right)\right)$.

Using this and $q_{1}>q_{2}$, we get, for any positive $k_{2}<k$,

$$
\begin{aligned}
1= & \left(S_{2}(k) / p_{1}\right)\left[\left(1+q_{1}+\ldots+q_{1}^{k_{2}-1}\right)+q_{1}^{k_{2}}\left(1+q_{1}+\ldots+q_{1}^{k-k_{2}-1}\right.\right. \\
\quad & \left.\quad+q_{1}^{k-k_{2}-1} q_{2}\left(1+q_{2}+\ldots+q_{2}^{s-k-1}\right)\right] \\
> & \left(S_{2}(k) / p_{1}\right)\left(\left(1+q_{1}+\ldots+q_{1}^{k_{2}-1}\right)+q_{1}^{k_{2}}\left(1+q_{2}+\ldots+q_{2}^{s-k_{2}-1}\right)\right) .
\end{aligned}
$$

Thus, $k$ is indeed the smallest integer for which (38) is satisfied. Therefore, $k$ can only increase, if the setup $S_{2}(k)$ is decreased sufficiently, otherwise $k$ would no longer be the minimum integer to keep the inequality valid.

Theorem 22 If $\left(p_{2}\right)^{2}>p_{1} p_{3}$, then the pattern-changing sublot $k$ can be found in $O(\log s)$ time.

Proof. Let $S_{\max }(k)$ be the maximum value of $S_{2}^{\prime}$ for which $k$ is the pattern-changing sublot. We show that $(2, k)-(3, k)-(3, k+1)$ is critical when $S_{2}^{\prime}=S_{\max }(k)$.

Suppose it is not, then by Theorem $15, x_{k+1}(k)>x_{k}(k) q_{2}=\left(S_{2}^{\prime} / p_{1}\right) q_{1}^{k-1} q_{2}$. Substituting into (37), we get

$$
\left(S_{2}^{\prime} / p_{1}\right)\left(1+q_{1}+\ldots+q_{1}^{k-1}+q_{1}^{k-1} q_{2}\left(1+q_{2}+\ldots+q_{2}^{s-k-1}\right)\right)<1 .
$$

If we increase $S_{2}^{\prime}$ a little, this inequality still holds for $k$, which implies that $k$ still is the pattern-changing sublot. This, however, contradicts the assumption of $S_{2}^{\prime}=S_{\max }(k)$.

Now we can do binary search in the interval $\left[1, k^{*}\right]$ for $k$, where $k^{*}$ is the pattern-changing sublot when $S_{2}^{\prime}=0$ [6]. Start with the trial value $k_{0}=\left\lceil k^{*} / 2\right\rceil$. By Theorem 20 and the fact that $\left(2, k_{0}\right)-\left(3, k_{0}\right)-\left(3, k_{0}+1\right)$ is critical if $S_{2}^{\prime}=S_{\max }\left(k_{0}\right)$, we have $S_{\max }\left(k_{0}\right)=p_{1} x_{1}\left(k_{0}\right), x_{i}\left(k_{0}\right)=x_{1}\left(k_{0}\right) q_{1}^{i-1}$ for $1 \leq i \leq k_{0}$ and $x_{j}\left(k_{0}\right)=x_{k}\left(k_{0}\right) q_{2}^{j-k_{0}}$ for $k_{0}<j \leq s$. Substituting into $\sum_{i=1}^{s} x_{i}\left(k_{0}\right)=1$, we can get $x_{1}\left(k_{0}\right)$ and $S_{\max }\left(k_{0}\right)$ in $O(1)$ time. If $S_{\max }\left(k_{0}\right)>$ $S_{2}^{\prime}$, then $k_{0}<k$, otherwise, $k_{0} \geq k$. Therefore, the total time required is $O(\log s)$. 
Remark 2. If $S_{2}^{\prime}>S_{\max }(1)$, then no sublot is critical on $M_{1}$ and the problem becomes a two-machine problem (on $M_{2}$ and $M_{3}$ ) with setup time $S_{2}^{\prime}$ on $M_{2}$ and 0 on $M_{3}$. It is clear, however, that the optimal sublot sizes for this two-machine problem are the same as for its relaxed version, i.e., when $S_{2}^{\prime}=0$.

By Theorem 20, $S_{\max }(1)=p_{1} x_{1}^{r}=p_{1} \frac{1-\left(p_{3} / p_{2}\right)}{1-\left(p_{3} / p_{2}\right)^{s}}$. Therefore, if $S_{2}^{\prime}>$ $p_{i} \frac{1-\left(p_{3} / p_{2}\right)}{1-\left(p_{3} / p_{2}\right)^{8}}$, then the original three-machine problem is equivalent to the relaxed problem on $M_{2}$ and $M_{3}$ with no setups.

\section{Summary and Concluding Remarks}

We have analyzed the structural properties of lot streaming schedules which minimize the makespan for a single job with detached setup times in an $m$ machine flow shop. The results of Glass et.al.[6], for the no-setup case, have been extended to the case of detached setups. For $m=2$ or 3 , we have proved that there is always an optimal schedule with consistent sublots.We have shown that the general problem is equivalent to one in which there is no setup time on the first and the last machine. Therefore, it is sufficient to consider a nonzero setup time $\left(S_{2}^{\prime}\right)$ only on the second machine when $m=3$. If $S_{2}^{\prime} \leq p_{1} x_{1}^{R}$, then the setup does not affect the optimality of the schedule without setups. However, if $S_{2}^{\prime}>p_{1} x_{1}^{R}$, then the setup time causes an increase in the makespan. We have proved that every sublot will be critical and will have a positive size for this case too.

The structure of the optimal network depends on the relative size of the job processing times on the three machines. When $\left(p_{2}\right)^{2}=p_{1} p_{3}$, the optimal solution is the same as with no setups, but none of the sublots stays critical on the first machine and the makespan is equal to $S_{2}^{\prime}$ plus the length of the optimal schedule with no setups for the two-machine problem on $M_{2}$ and $M_{3}$. When $\left(p_{2}\right)^{2} \neq p_{1} p_{3}$, the optimal schedule and its structure change substantially in comparison with the no-setup case: The optimal network can be characterized by a combination of two patterns with a patternchanging sublot between them. The optimal sublot sizes follow one geometric progression up to the pattern-changing sublot and another one after it. The index of the pattern-changing sublot depends on the setup. Each index $k$ remains valid in an interval of setup times and changes in a monotone fashion with the setup time, but in different directions, depending on whether 
$\left(p_{2}\right)^{2}<p_{1} p_{3}$ or $\left(p_{2}\right)^{2}>p_{1} p_{3}$. Exploiting this monotonicity has resulted in $O(\log s)$ time computations of the optimal schedule.

In certain situations it is desirable to have no-wait schedules, i.e., to be able to start the processing of each sublot on each machine immediately after it is finished on the preceding machine. It can be easily checked from the structure of the optimal networks that, similarly to the no-setup case [6], the no-wait requirement can be satisfied without increasing the length of the optimal schedule.

Sometimes no-idling may be required, i.e., each machine should be kept working without any idle time once it starts. (This was called the contiguity of work assumption in [17] and in [18].) It is straightforward to transform the optimal schedules into a no-idling schedule on the first and last machine. The situation is more complicated, however, on the second machine. When $\left(p_{2}\right)^{2}=p_{1} p_{3}$ or when $\left(p_{2}\right)^{2}>p_{1} p_{3}$, then every horizontal segment of the network is critical on $M_{2}$, and the no-idling requirement is satisfied. When $\left(p_{2}\right)^{2}<p_{1} p_{3}$, however, a no-idling schedule will have an increased makespan.

There are many related topics for future investigation. It seems to be natural to try to extend the results in this paper to the case of job shops and open shops. The analysis of the lot streaming problem in a flow shop with attached setup times on the machines is another possibility. Some of these questions will be studied in the future.

\section{References}

[1] Baker, K.R., "Elements of Sequencing and Scheduling", K.R. Baker publ., Hanover, NH 1992.

[2] Baker, K.R., "Lot Streaming in the Two-Machine Flow Shop with Setup Times", Working Paper No. 297, A. Tuck School of Bus. Admin., Dartmouth College, Hanover NH, 1993.

[3] Cetinkaya, F.C., and Gupta, J.N.D., "Flowshop Lot Streaming to Minimize Total Weighted Flow Time", Working Paper No. 94-24, Industrial Engineering, Purdue Univ., West Lafayette, IN, 1994. 
[4] Cetinkaya, F.C., and Kayaligil, M.S., "Unit Sized Transfer Batch Scheduling with Setup Times", Computers and Industrial Engineering, 22 (1992) 177-183.

[5] Fox, R.E., "OPT - An Answer for America (Part IV)", Inventories and Production 3 (1983).

[6] Glass, C.A., Gupta, J.N.D, and Potts, C.N., "Lot Streaming In Three Stage Production Processes", Europ. J. Oper. Research, 75 (1994) 378394.

[7] Goldratt, E., and Fox, R.E., The Race, North River Press, Croton-onHudson, NY, 1986.

[8] Goyal, S.K., "Note on Manufacturing Cycle Time Determination for a Multi-Stage Economic Production Model", Management Science 23 (1976) 332-333; the rejoinder, 334-338.

[9] Graves, S.C., and Kostreva, M., "Overlapping Operations in Material Requirements Planning", J. Operations Management 6 (1987) 283-294.

[10] Jacobs, F.R., and Bragg, D.J., "Repetitive Lots: Flow Time Reductions Through Sequencing and Dynamic Batch Sizing", Decision Sciences 19 (1988) 281-294.

[11] Kulonda, D.J., "Overlapping Operations - A Step Towards Just-In-Time Production", Readings in Zero Inventory, APICS 27th Annual Conf. (1984) 78-80.

[12] Moily, J.P., "Optimal and Heuristic Procedures for Component LotSplitting in a Flow Shop", Management Science 32 (1986) 113-125.

[13] Monma, C.L., and Rinnooy Kan, A.H.G., "A Concise Survey of Efficiently Solvable Special Cases of the Permutation Flow-Shop Problem", RAIRO Recherche Opérationelle 17(1983) 105-119.

[14] Potts, C.N., and Baker, K.R.,"Flow Shop scheduling With Lot Streaming", Operations Research Letters 8 (1989) 207 - 303. 
[15] Potts, C.N., and Van Wassenhove, L.N., "Integrating Scheduling With Batching and Lot-Sizing : a Review of Algorithms and Complexity", $J$. Oper. Res. Soc. 43 (1992) 395-406.

[16] Reiter, S., "A System for Managing Job Shop Production", J. of Business, 34 (1966) 371-393.

[17] Szendrovits, A.Z. "Manufacturing Cycle Time Determination for a Multi-Stage Economic Production Quantity Model", Management Science, 22 (1978), 298-308.

[18] Steiner, G., and Truscott, W.G., "Batch Scheduling to Minimize Cycle Time, Flow Time, and Processing Cost", IIE Transactions, 23 (1993) 90-97.

[19] Trietsch, D., "Optimal Transfer Lots For Batch Manufacturing : A Base Case And Extension", Technical Report NPS-54_87-010, Naval Postgraduate School, Monterey, CA, 1987.

[20] Trietsch, D., " Polynomial Transfer Lot Sizing Techniques For Batch Processing On Consecutive Machines", Technical Report NPS-54-89011, Naval Postgraduate School, Monterey, CA, 1989.

[21] Trietsch, D., and Baker, K.R., "Basic Techniques For Lot Streaming", Operations Research, 41 (1993), 1065-1076.

[22] Vickson, R. "Optimal Lot Streaming For Multiple Products in a TwoMachine Flow Shop", Working paper, Department of Management Sciences, Univ. Waterloo, Canada, 1992, to appear Europ. J. Oper. Res.

[23] Vickson, R., and Alfredsson, B., "Two and Three Machines Flow Shop Scheduling Problems With Equal Sized Transfer Batches", Int. J. Production Res. 30 (1992) 1551-1574. 


\section{School of Business \\ McMaster University \\ WORKING PAPERS - RECENT RELEASES}

364. Robert F. Love, John H. Walker, and Moti L. Tiku, "Confidence Levels for $\ell_{k, p, \theta}$ Distances", June, 1991.

365. Archer, N. P., and G. 0. Wesolowsky, "A Dynamic Service Quality Cost Model with Word-of-Mouth Advertising", July, 1991.

366. John W. Medcof, "Weiner, Kelley, Jones, and Davis, Peat: Integrated", Aúgust, 1991.

367. Harish C. Jain and Rick D. Hackett, "A Comparison of Employment Equity and Non-Employment Equity Organizations On Designated Group Representation And Views Towards Staffing", August, 1991.

368. J. Brimberg and R. F. Love, "Local Convergence in a Generalized Fermat-Weber Probiem", August, 1991.

369. Rick D. Hackett and Peter Bycio, "The Temporal Dynamics Associated with Absenteeism as a Coping Mechanism", September, 1991.

370. Robert F. Love and John H. Walker, "A New Criterion For Evaluating the Accuracy of Distance Predicting Functions", October, 1991.

371. Joseph B. Rose, "Interest and Rights Disputes Resolution in Canada, New Zealand and Australia", October, 1991.

372. Paul A. Dion and Peter M. Banting, "Buyer Reactions to Product Stockouts in Business to Business Markets", October, 1991.

373. Isik U. Zeytinoglu, "Part-Time and Other Non-Standard Forms of Employment: Why are They Considered Appropriate for Women?", November, 1991.

374. Rick D. Hackett, Peter Bycio and Peter Hausdorf, "Further Assessments of a Three-Component Model of Organizational Commitment", January, 1992.

375. Y. Lilian Chan, Bernadette E. Lynn, "Evaluating Tangibles and Intangibles of Capital Investments", February, 1992.

376. Christopher K. Bart, "Strategic Lessons for Today's Airline CEO's", February, 1992.

377. Yufei Yuan, "A Stable Residence Exchange Problem", March, 1992.

378. Jim Gaa, "The Auditor's Role: The Philosophy and Psychology of Independence and Objectivity". Apri1, 1992. 
379. David J. Buchanan and George 0. Wesolowsky, "Algorithm for Rectilinear Distance Minimax Single Facility Location in the Presence of Barriers to Travel", Apri1, 1992.

380. Thomas E. Muller and D. Wayne Taylor, "Eco-Literacy Among Consumers: How Much Do They Know About Saving Their Planet?", Apri1, 1992.

381. Willi $\mathrm{H}$. Wiesner, "The Contributions of Job Relevance, Timing, and Rating Scale to the Validity of the Structured Employment Interview", September, 1992.

382. Robert G. Cooper, "Third Generation New Product Processes", November, 1992.

383. Robert F. Love and John H. Walker, "Distance Data for Eighteen Geographic Regions", August, 1993.

384. Jack Brimberg and Robert F. Love, "New Hull Properties for Location Problems", August, 1993

385. Harish C. Jain and S. Muthuchidambaram, "Bi11 40 Amendments to Ontario Labour Relations Act: An Overview and Evlauation", September, 1993.

386. Norm P. Archer and Randy Bassett, "Self-Organizing Feature Maps: The Traveling Salesman Problem and Other Applications". September, 1993.

387. John W. Medcof, "Need Satisfaction and Job Behaviours in Four Occupations", October, 1993.

388. Harish C. Jain and C.S. Venkata Ratnam, "Affirmative Action in Employment for the Scheduled Castes and the Scheduled Tribes in India", December, 1993.

389. Harish C. Jain and S. Muthu, "A Legislative Framework Restricting the Use of Replacement Workers: The Ontario Approach and Its Relevance to U.S. Labor Law Reform", December, 1993.

390. N.P. Archer, M.M. Sigmund, J.P. Wollersheim and Y. Yuan, "Interface Support for Information Search in Decision Making: Effects of Voice and Text Output Modes with Information Abstraction", January, 1994.

391. Suzanne C. Grunert and Thomas E. Muller, "Measuring Values in International Settings: Are Respondents Thinking "Real" Life or "Ideal" Life?, February, 1994.

392. Thomas E. Muller, "Values as Determinants of Experience-Based Affect in International Tourists", March, 1994.

393. Naresh C. Agarwal, "Mandatory vs. Flexible Retirement in Canada: Law, Issues and Policy Implications", March, 1994. 
394. Joseph B. Rose and Michael Piczak, "Settlement Rates and Settlement Stages", August, 1994.

395. Roy Adams, "A Pernicious Euphoria: 50 Years of Wagnerism in Canada", November, 1994.

396. Roy Adams, "The Determination of Trade Union Representativeness in Canada and the United States", November, 1994.

397. Shouhong Wang, Norman P. Archer, "A Fuzzy Decision Making Model", December, 1994.

398. Kalyan Moy Gupta and A7i R. Montazemi, "A Methodology for Evaluating the Retrieval Performance of Case-Based Reasoning Systems", December, 1994. 
Innis Ref.

AB

74.5

.247

ro. 399

1258992 